\title{
Workshop Chairs' Welcome
}

It is our great pleasure to welcome you to the WWW 2018 Workshops. This year's workshops of WWW 2018 feature a number of co-located workshops that are intended to provide a forum for researchers and practitioners in Web technologies to discuss and exchange positions on current and emergent Web topics. We received forty proposals from all around the world covering a broad range of topics. We evaluated them regarding relevance, quality, and novelty selecting eighteen fullday workshops and ten half-day workshops. We also took into account the coverage of the different areas related to WWW as well as the potential audience, to schedule them in two consecutive days with the minimal audience interest overlap.

The first day includes the following twelve workshops

- 8 8th Temporal Web Analytics Workshop (TempWeb)

Organizers: Marc Spaniol, Ricardo Baeza-Yates and Julien Masanes

- Enhancing Citizen Centricity with Web Applications (4th AW4city)

Organizers: Leonidas Anthopoulos, Marijn Janssen and Vishanth Weerakkody

- Online Recommender Systems and User Modeling (ORSUM)

Organizers: Alipio M. Jorge, João Vinagre, Myra Spiliopoulou and Pawel Matuszyk

- Exploitation of Social Media for Emergency Relief and Preparedness

Organizers: Marie-Francine Moens, Gareth Jones, Saptarshi Ghosh, Debasis Ganguly,

Kripabandhu Ghosh and Tanmoy Chakraborty

- Social Sensing and Enterprise Intelligence : Towards a Smart Enterprise Transformation Organizers: Lipika Dey, Tirthankar Dasgupta and Priyanka Sinha

- Extreme Multilabel Classification for Social Media Organizers: Akshay Soni, Robert Busa-Fekete, Krzysztof Dembczyński and Aasish Pappu

- 11th International Workshop on Linked Data on the Web Organizers: Tim Berners-Lee, Sarven Capadisli, Stefan Dietze, Aidan Hogan, Krzysztof Janowicz and Jens Lehmann

- $\quad$ 1st International Workshop on Hybrid Question Answering with Structured and Unstructured Knowledge (HQA'18) Organizers: Franz Baader, Brigitte Grau and Yue Ma

- Educational Knowledge Management workshop (3rd edition) Organizers: Inaya Lahoud, Elsa Cardoso and Nada Matta

- 9th International Workshop on Web APIs and Service Architecture (WS-REST) Organizers: Erik Wilde, Mike Amundsen and Mehdi Medjaoui

- 6th International Workshop on Natural Language Processing for Social Media (SocialNLP) Organizers: Lun-Wei Ku and Cheng-Te Li

- Mining Attributed Networks Workshop Organizers: Martin Atzmueller, Sabrina Gaito, Roberto Interdonato, Rushed Kanawati, Christine Largeron, Matteo Magnani, and Alessandra Sala 
The second day includes the following fifteen workshops

- 8th International Workshop on Location and the Web (LocWeb)

Organizers: Dirk Ahlers, Erik Wilde, Rossano Schifanella, Jalal S. Alowibdi and Muhammad Zubair Shafiq

- International Workshop on Profiling and Searching Data on the Web (PROFILES \& Data:Search)

Organizers: Laura Koesten, Elena Demidova, Vadim Savenkov, John Breslin, Oscar Corcho, Stefan Dietze and Elena Simperl

- Web Stream Processing

Organizers: Payam Barnaghi, Jean-Paul Calbimonte and Daniele Dell'Aglio

- \#RCBlackMirror2018: Re-Coding Black Mirror

Organizers: Pinelopi Troullinou, Mathieu D’Aquin and Ilaria Tiddi

- Semantics, Analytics and Visualisation: Enhancing Scholarly Dissemination (SAVE-SD)

Organizers: Alejandra Gonzalez-Beltran, Francesco Osborne, Silvio Peroni and Sahar

Vahdati

- Wiki Workshop

Organizers: Robert West, Leila Zia, Dario Taraborelli and Jure Leskovec

- Researcher Centric Scholarly Communication

Organizers: Sarven Capadisli, Herbert Van de Sompel and Wendy Hall

- $\quad 3 r d$ Workshop on Linked Data \& Distributed Ledgers (LD-DL)

Organizers: Luis-Daniel Ibanez, John Domingue and Pascal Molli

- RoD: Reasoning on Data

Organizers: Marie-Laure Mugnier, Catherine Roussey and Pierre Senellart

- Augmenting Intelligence with Humans-in-the-Loop (HumL)

Organizers: Lora Aroyo, Gianluca Demartini, Anna Lisa Gentile and Chris Welty

- Second Women in Data Science Workshop (WinDS)

Organizers: Ana Paula Appel, Marisa Vasconcelos, Francesca Spezzano and Leman Akoglu

- 3rd International Workshop on Learning Representations for Big Networks (BigNet)

Organizers: Jie Tang, Michalis Vazirgiannis, Yuxiao Dong and Fragkiskos Malliaros

- $\quad$ 9th International Workshop on Modeling Social Media (MSM): Applying Machine Learning and AI for Modeling Social Media

Organizers: Martin Atzmueller, Alvin Chin and Christoph Trattner

- 3rd International Workshop on Computational Methods for CyberSafety

Organizers: Richard Han, Jeremy Blackburn and Homa Hosseinmardi

- Online Social Networks and Media: Network Properties and Dynamics (ONSED)

Organizers: Remy Cazabet, Giulio Rossetti and Esteban Bautista 
We believe that the program provides a good balance between several trending topics such as data parallelization, social media analysis, graph mining, crowdsourcing, knowledge databases, semantic data management, distributed web caching, humans-in-the-loop, recommendation systems, large-scale distributed machine learning, mobile data, antisocial behavior, among others. We hope that you will find the workshop program interesting, providing you with a valuable opportunity to learn and share ideas with other researchers and practitioners from institutions around the world.

Eyhab Al-Masri

Workshop Co-Chair

University of Washington

Marie-Christine Rousset

Workshop Co-Chair

Universite Grenoble Alpes

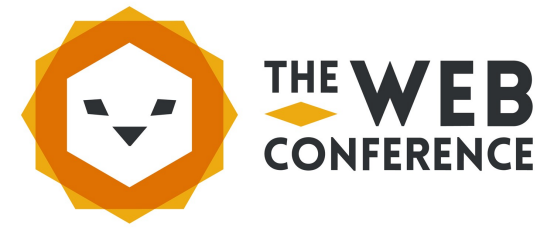

